\title{
Mutation Analysis of MYORG in a Chinese Cohort With Primary Familial Brain Calcification
}

\author{
Yi-Heng Zeng ${ }^{\dagger}$, Bi-Wei Lin ${ }^{\dagger}$, Hui-Zhen Su, Xin-Xin Guo, Yun-Lu Li, Lu-Lu Lai, Wan-Jin Chen*, \\ Miao Zhao* and Xiang-Ping Yao*
}

Department of Neurology and Institute of Neurology of First Affiliated Hospital, Institute of Neuroscience, and Fujian Key Laboratory of Molecular Neurology, Fujian Medical University, Fuzhou, China

\section{OPEN ACCESS}

Edited by:

João Ricardo Mendes Oliveira, Federal Rural University of

Pernambuco, Brazil

Reviewed by:

Gaël Nicolas,

Centre Hospitalier Universitaire (CHU)

de Rouen, France

Eriton Cunha,

Federal University of Pernambuco,

Brazil

*Correspondence:

Wan-Jin Chen

waniinchen75@fjmu.edu.cn

Xiang-Ping Yao

yaoxiangping@fjmu.edu.cn

Miao Zhao

zhaomiao91@fjmu.edu.cn

${ }^{\text {t}}$ These authors have contributed equally to this work

Specialty section:

This article was submitted to

Genetics of Common and Rare

Diseases,

a section of the journal

Frontiers in Genetics

Received: 29 June 2021

Accepted: 09 September 2021

Published: 18 October 2021

Citation:

Zeng $Y-H$, Lin B-W, Su H-Z, Guo X-X,

Li Y-L, Lai L-L, Chen W-J, Zhao M and Yao X-P (2021) Mutation Analysis of MYORG in a Chinese Cohort With

Primary Familial Brain Calcification.

Front. Genet. 12:732389.

doi: 10.3389/fgene.2021.732389
Primary familial brain calcification (PFBC) is a progressive neurological disorder manifesting as bilateral brain calcifications in CT scan with symptoms as parkinsonism, dystonia, ataxia, psychiatric symptoms, etc. Recently, pathogenic variants in MYORG have been linked to autosomal recessive PFBC. This study aims to elucidate the mutational and clinical spectrum of MYORG mutations in a large cohort of Chinese PFBC patients with possible autosomal recessive or absent family history. Mutational analyses of MYORG were performed by Sanger sequencing in a cohort of $245 \mathrm{PFBC}$ patients including 21 subjects from 10 families compatible with a possibly autosomal-recessive trait and 224 apparently sporadic cases. Indepth phenotyping and neuroimaging features were investigated in all patients with novel MYORG variants. Two nonsense variants (c.442C > T, p. Q148*; c.972C > A, p. Y324*) and two missense variants (c.1969G >C, p. G657R; c.2033C > G, p. P678R) of MYORG were identified in four sporadic PFBC patients, respectively. These four novel variants were absent in gnomAD, and their amino acid were highly conserved, suggesting these variants have a pathogenic impact. Patients with MYORG variants tend to display a homogeneous clinical spectrum, showing extensive brain calcification and parkinsonism, dysarthria, ataxia, or vertigo. Our findings supported the pathogenic role of MYORG variants in PFBC and identified two pathogenic variants (c.442C > T, c.972C > A), one likely pathogenic variant (c.2033C > G), and one variant of uncertain significance (c.1969G>C), further expanding the genetic and phenotypic spectrum of PFBC-MYORG.

Keywords: primary familial brain calcification, MYORG, mutations, parkinsonism, phenotype

\section{INTRODUCTION}

Primary familial brain calcification (PFBC), widely known as Fahr's disease, is a rare inherited neurodegenerative disease characterized by bilateral calcium deposits in the basal ganglia and/or other brain regions, in the absence of other secondary causes for brain calcification (Manyam, 2005). Affected individuals can exhibit a wide range of clinical symptoms, including dystonia, parkinsonism, ataxia, cognitive impairment, and psychiatric symptoms, while some remain asymptomatic for their entire lives (Nicolas et al., 2013a). The prevalence of PFBC is still unknown, and some studies indicate that it is underdiagnosed because of its unspecific presentations (Nicolas et al., 2018). 
Typically, PFBC is inherited in an autosomal dominant manner (AD-PFBC); to date, four autosomal dominant PFBC-associated genes have been identified, including SLC20A2, PDGFRB, PDGFB, and XPR1 (Wang et al., 2012; Nicolas et al., 2013b; Keller et al., 2013; Legati et al., 2015). Little is known about their mechanisms, which are thought to be related to phosphate homeostasis via mutations in SLC20A2 and XPR1, and pericyte function affecting the blood-brain barrier integrity by mutations in $P D G F R B$ and $P D G F B$. Our study demonstrated that SLC20A2 accounted for the highest contribution (14.2\%) in Chinese PFBC, followed by $P D G F R B, P D G F B$, and XPR1 ( $0.9 \%$ each) (Guo et al., 2019). As such, a substantial proportion of patients remain genetically undiagnosed (Taglia et al., 2015). Recently, we have first reported disease-causing mutations in MYORG gene (MIM: 618255) for the autosomal recessive form of PFBC (AR-PFBC) (MIM \#618317) (Yao et al., 2018). Another team identified JAM2 as a gene related to autosomal recessive $\mathrm{PFBC}$, further supporting the recessive pathogenic gene as a factor in PFBC pathogenesis (Cen et al., 2020).

There is currently little information about the mutational and clinical spectrum of Chinese patients with PFBC. In the present study, we analyze detailed genetic and clinical data from four PFBC patients carrying novel MYORG variants and investigate the relationship between mutations, phenotyping, and neuroimaging features.

\section{MATERIAL AND METHODS}

\section{Subjects}

A total of 245 patients with possible autosomal recessive traits or negative family history were recruited from multiple hospitals in China from January 2012 to August 2021, which had excluded the previously reported recessive families (Yao et al., 2018). The criteria for the diagnosis of PFBC were as follows: 1) bilateral and symmetrical calcifications in the basal ganglia and/or dentate nucleus detected by CT scans; 2) a total calcification score (TCS) rates above the age-specific thresholds (Nicolas et al., 2013a); 3) absence of biochemical abnormalities, including serum concentration of calcium, phosphate, and parathyroid hormone; 4) secondary causes of brain calcification were excluded such as infectious, toxic, or traumatic causes. All sporadic patients were previously tested for variants of all $A D-P F B C$ genes by Sanger sequencing, and no pathogenic variants were detected (SLC20A2, PDGFRB, PDGFB, and $X P R 1)$. For all patients, a complete neurological examination was performed by two neurologists. The calcifications in the cerebral locations were further evaluated with TCS (range from 0 to 80 ). In cases with biallelic MYORG variants, previous investigations were retrospectively analyzed based on a chart review where available: neuroimaging in all reported cases with CT $(n=$ $3)$ and brain MRI $(n=1)$. We also recruited 200 individuals without brain calcification as normal controls. The study was approved by the institutional review board at the First Affiliated Hospital of Fujian Medical University. All subjects provided informed consent before inclusion.

\section{Mutational Analysis}

We collected venous blood samples from all participants including all available family members. Genomic DNA was extracted from peripheral blood leukocytes using standard protocols. Polymerase chain reaction (PCR) was performed to amplify the coding exon (exon 2) of the MYORG gene (NM_020702.5), using the primers previously reported (Yao et al., 2018). The PCR products were purified and analyzed by Sanger sequencing with ABI 3730XL automated DNAsequencing system. The sequence data were further analyzed and compared with reference MYORG coding sequences from the Human Genome database (NM_020702.5).

The identified variants that fulfilled the following criteria were included for further analysis: 1) excluded variants with frequencies exceeding $0.1 \%$ in the 1,000 Genomes Project (www.1000genomes.org), Exome Aggregation Consortium (ExAC, http://exac.broadinstitute.org/), or Genome Aggregation Database (gnomAD v2.1.1; http://gnomad. broadinstitute.org); 2) excluded synonymous variants and missense variants, which were predicted to be nonpathogenic in silico by Mutation Taster (http://www.mutationtaster.org/), Polyphen2 (http://genetics.bwh.harvard.edu/pph2/), or SIFT (http://sift.jcvi.org/). The evolutionary conservation of the affected amino acid among different species was estimated using HomoloGene (http://www.ncbi.nlm.nih.gov/ homologene). All novel variants were independently classified by two investigators based on the American College of Medical Genetics and Genomics (ACMG) guidelines and the Association for Molecular Pathology (Richards et al., 2015).

\section{RESULTS}

\section{MYORG Variants in the Primary Familial Brain Calcification Cohort}

We screened a total of 245 Chinese subjects including 21 subjects from 10 possibly autosomal recessive families and 224 sporadic cases. Eight MYORG variants were identified in six sporadic cases, while four of the eight variants were previously reported (c.103A > G, p.M35V; c.782_783GC > TT, p. R261L; c.1092_1097delCTTCGA, p.365_366delFD; and c.1967T > C, p. I656T) (Supplementary Figures S1A-D) (Yao et al., 2018; Forouhideh et al., 2019; Chelban et al., 2020; Chen et al., 2020). Notably, four novel variants in MYORG were identified: two nonsense variants (c.442C > T, p. Q148*; c.972C > A, p. Y324*) and two missense variants (c.1969G>C, p. G657R; c.2033C > G, p. P678R) (Figures 1A-D). The variants, c.442C > T and c.2033C > G, were separately found in a homozygous state in case 1 and case 4 , while c.972C > A and c.1969G $>$ C were identified in a heterozygous state in case 2 and case 3 , respectively coupled with the previously reported variants (c.103A > G and c.782_783GC > TT) (Table 1). 
A

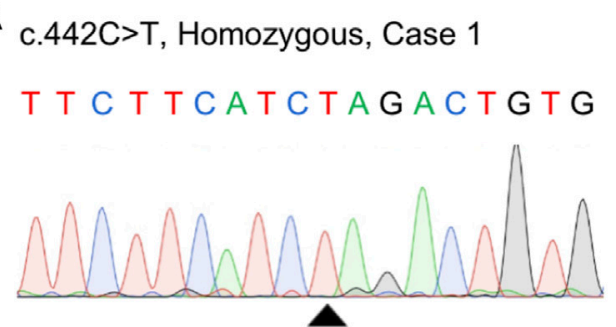

T T C T TCATCCAGACTGTG

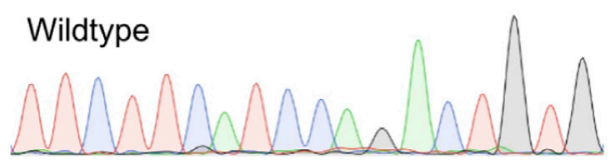

C

c. $1969 \mathrm{G}>\mathrm{C}$, Heterozygous, Case 3

G T T C C T TAT T CG GG ACACGC

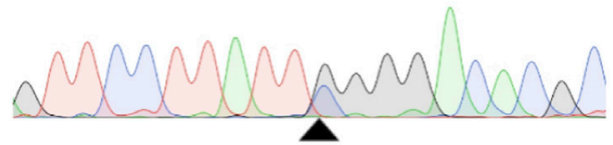

G T TCCT TAT T GGGGACACGC

Wildtype

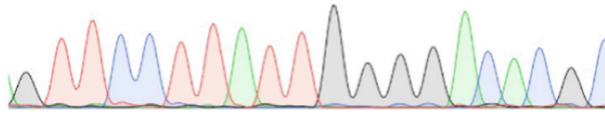

B c. $972 \mathrm{C}>\mathrm{A}$, Heterozygous, Case 2

G G C GC TG T A A G G G C C G C C

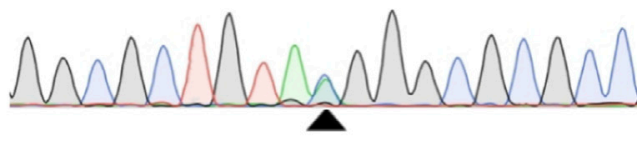

G G C GC TG TACG G G G C G C C

Wildtype

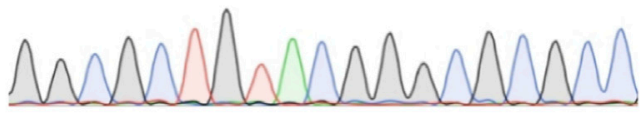

D

c.2033C>G, Homozygous, Case 4

T C T A T T T G C G C G C C G G C A

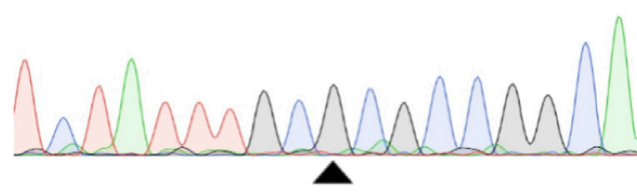

T C T A T T T G C C C G C C G G C A

Wildtype

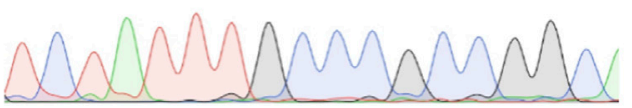

FIGURE 1 | Identification of four novel variants in our study. (A-D) Sanger sequences of the MYORG variants in the four cases and corresponding wild-type subjects.

\section{Evaluation of the Pathogenicity of the Three MYORG Missense Variants}

None of the novel variants mentioned above were present in the 1000G, ExAC, or gnomAD (Table 1). In silico analysis predicted deleterious consequences using the Mutation Taster, SIFT, Polyphen2, and CADD software programs. The two missense variants were both located at the highly conserved positions and the glycosidase domain (Figure 2A). Based on the American College of Medical Genetics (ACMG) guidelines, the two nonsense variants were considered as "Pathogenic;" the missense variant c.2033C > G was considered as "Likely Pathogenic;" and c.1969G>C was considered as "Variants of Uncertain Significance" (Table 1). Four of the 224 sporadic PFBC (1.79\%) carried variants in MYORG in this study.

\section{Clinical Manifestations of the Patients Carrying Novel MYORG Mutations}

The clinical manifestations of the patients with novel MYORG variants are summarized in Table 1. All four patients showed extensive calcification involving the basal ganglia, dentate nuclei, thalamus, and the subcortex in CT/MRI scans. Patients displayed a homogeneous clinical pattern and commonly experienced parkinsonism, dysarthria, ataxia, and vertigo. Detailed clinical and radiological findings of the patients are described as follows.

Case 1 . The patient with a c.442C > T nonsense homozygous variant was a 73-year-old man who was admitted to the hospital because of dysarthria, gait impairment, and vertigo. These symptoms were experienced for 7 years. There was an initial progressive slurred speech, walked unsteadily, starting hesitantly with reduced up and down gaze, associated with profound bilateral bradykinesia, and a combination of ataxia and freezing. Progressive deterioration of dysarthria and bradykinesia became evident over the following years. He had difficulty in tandem walking and could not walk independently. A neurological examination revealed cerebellar dysarthria, supranuclear gaze palsy, bradykinesia, festinating gait, and mild rigidity in the lower limbs; no tremor was observed. His cerebral MRI at age 72 years showed high-intensity in the globus pallidus, thalamus, dentate nuclei, and subcortical white matter, suggesting moderate symmetrical calcification (Figures $2 \mathbf{B a}-\mathbf{c}$ ). The patient reported no family history of brain calcification.

Case 2. The patient, carrying c.972C > A and c.103A > G heterozygous variants of $M Y O R G$, was a 47-year-old woman who had slurred speech with slow progression. She later developed dysphagia and forced laughter or crying. No other abnormalities 
TABLE 1 | Clinical features in PFBC patients with MYORG mutations F, female; M, male; AAE, age at examination; AAO, age at onset; y, years; TCS, total calcification score; NA, not available.

\begin{tabular}{|c|c|c|c|c|c|c|c|c|c|c|c|c|c|c|c|}
\hline \multirow{2}{*}{$\begin{array}{l}\text { Case } \\
\text { No }\end{array}$} & \multirow[t]{2}{*}{ Sex } & \multirow{2}{*}{$\begin{array}{l}\text { AAE } \\
\text { (y) }\end{array}$} & \multirow{2}{*}{$\begin{array}{l}\text { AAO } \\
\text { (y) }\end{array}$} & \multirow{2}{*}{$\begin{array}{l}\text { Clinial } \\
\text { features }\end{array}$} & \multicolumn{2}{|c|}{ Calcification } & \multirow[t]{2}{*}{ Zygosity } & \multirow{2}{*}{$\begin{array}{c}\text { CDNA } \\
\text { alteration }\end{array}$} & \multirow{2}{*}{$\begin{array}{l}\text { Amino } \\
\text { acid } \\
\text { alteration }\end{array}$} & \multirow{2}{*}{$\begin{array}{l}\text { GnomAD } \\
\text { frequency } \\
\text { (global) }\end{array}$} & \multirow{2}{*}{$\begin{array}{c}\text { Mutation } \\
\text { Taster }\end{array}$} & \multirow[t]{2}{*}{ SIFT } & \multirow{2}{*}{$\begin{array}{c}\text { PolyPhen } \\
-2\end{array}$} & \multirow[t]{2}{*}{ CADD } & \multirow{2}{*}{$\begin{array}{c}\text { ACMG } \\
\text { Classification }\end{array}$} \\
\hline & & & & & Localization & TSC & & & & & & & & & \\
\hline 1 & M & 72 & 66 & $\begin{array}{l}\text { dysarthria, } \\
\text { parkinsonism, } \\
\text { vertigo }\end{array}$ & $\begin{array}{l}\text { globus } \\
\text { pallidus, } \\
\text { thalamus, } \\
\text { dentate } \\
\text { nuclei, } \\
\text { subcortical } \\
\text { white matter }\end{array}$ & 18 & homozygous & c. $442 \mathrm{C}>\mathrm{T}$ & p.Q148* & absent & $\begin{array}{l}\text { Disease } \\
\text { causing }\end{array}$ & NA & NA & 36.0 & $\begin{array}{l}\text { Pathogenic } \\
\text { PVS1+PM2+PM3+PP3 }\end{array}$ \\
\hline \multirow[t]{2}{*}{2} & \multirow[t]{2}{*}{$\mathrm{F}$} & \multirow[t]{2}{*}{47} & \multirow[t]{2}{*}{42} & \multirow{2}{*}{$\begin{array}{l}\text { dysarthria, } \\
\text { dysphagia, } \\
\text { forced laughter } \\
\text { or crying }\end{array}$} & \multirow{2}{*}{$\begin{array}{l}\text { basal ganglia, } \\
\text { thalamus, } \\
\text { dentate } \\
\text { nuclei, } \\
\text { subcortical } \\
\text { white matter }\end{array}$} & \multirow[t]{2}{*}{43} & \multirow[t]{2}{*}{ heterozygous } & c. $972 \mathrm{C}>\mathrm{A}$ & p.Y324* & absent & $\begin{array}{l}\text { Disease } \\
\text { causing }\end{array}$ & NA & NA & 34.0 & $\begin{array}{l}\text { Pathogenic } \\
\text { PVS1+PM2+PP3 }\end{array}$ \\
\hline & & & & & & & & c. $103 \mathrm{~A}>\mathrm{G}$ & p.M35V & 0.000289 & $\begin{array}{l}\text { Disease } \\
\text { causing }\end{array}$ & Tolerated & $\begin{array}{l}\text { Probably } \\
\text { damaging }\end{array}$ & 24.0 & $\begin{array}{l}\text { Likely pathogenic } \\
\text { PS1+PM2+PP3+PP5 }\end{array}$ \\
\hline \multirow[t]{2}{*}{3} & \multirow[t]{2}{*}{$\mathrm{F}$} & \multirow[t]{2}{*}{68} & \multirow[t]{2}{*}{45} & \multirow[t]{2}{*}{ vertigo } & $\begin{array}{l}\text { basal ganglia, } \\
\text { dentate } \\
\text { nuclei, }\end{array}$ & \multirow[t]{2}{*}{ NA } & \multirow[t]{2}{*}{ heterozygous } & c. $1969 G>C$ & p.G657R & absent & $\begin{array}{l}\text { Disease } \\
\text { causing }\end{array}$ & Damaging & $\begin{array}{l}\text { Probably } \\
\text { damaging }\end{array}$ & 28.9 & $\begin{array}{l}\text { Variants of uncertain } \\
\text { significance } \\
\text { PM1+PM2+PP3 }\end{array}$ \\
\hline & & & & & $\begin{array}{l}\text { thalamus, } \\
\text { corona } \\
\text { radiata }\end{array}$ & & & c.782_783 GC> $>T$ & p.R261L & absent & $\begin{array}{l}\text { Disease } \\
\text { causing }\end{array}$ & Tolerated & Benign & NA & $\begin{array}{l}\text { Likely pathogenic } \\
\text { PS1+PM2+PP5 }\end{array}$ \\
\hline 4 & M & 62 & 59 & $\begin{array}{l}\text { dysarthria, } \\
\text { parkinsonism, } \\
\text { ataxia }\end{array}$ & $\begin{array}{l}\text { basal ganglia, } \\
\text { thalamus, } \\
\text { dentate } \\
\text { nuclei, } \\
\text { subcortical } \\
\text { white matter, } \\
\text { cerebellar } \\
\text { vermis, } \\
\text { brainstem }\end{array}$ & 57 & homozygous & c. $2033 \mathrm{C}>\mathrm{G}$ & p.P678R & absent & $\begin{array}{l}\text { Disease } \\
\text { causing }\end{array}$ & Damaging & $\begin{array}{l}\text { Probably } \\
\text { damaging }\end{array}$ & 28.3 & $\begin{array}{l}\text { Likely pathogenic } \\
\text { PM1+PM2+PM3+ } \\
\text { PP3+PP4 }\end{array}$ \\
\hline
\end{tabular}


A Organism
H. saplens
P.troglodytes
M. musculus
G. gallus
B T. rubripes

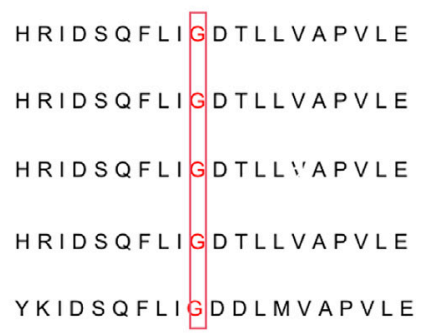

p.G657R
p.P678R

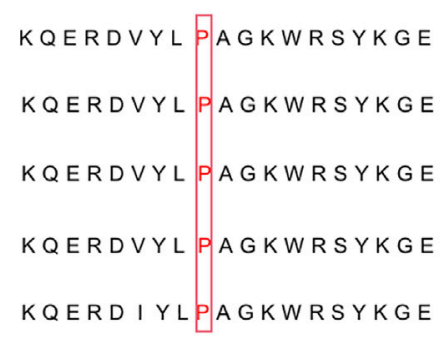

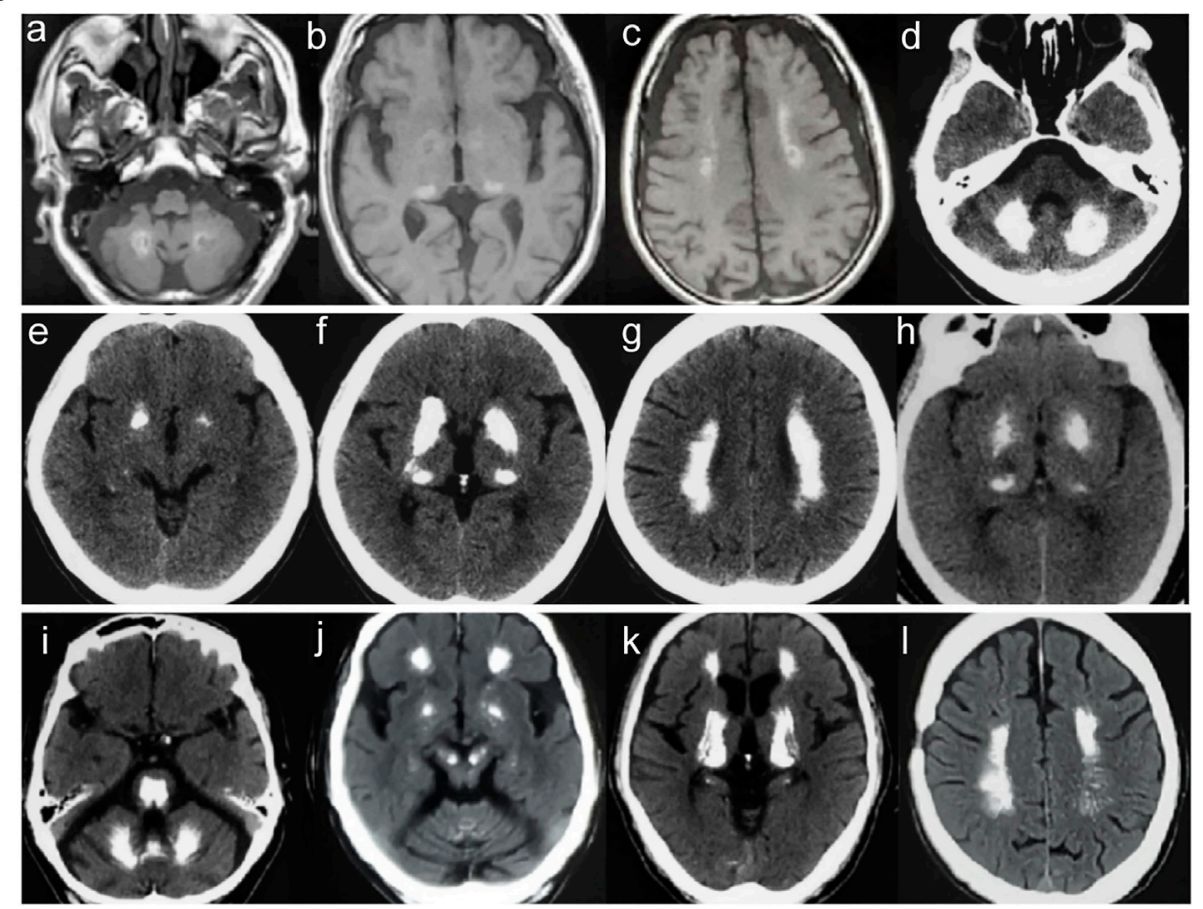

FIGURE 2 | (A) Conservation analysis across species for the novel missense MYORG variants. The variants were marked with red boxes for the corresponding amino acid. (B) Neuroimaging spectrum in the four cases. Cerebral MRI of Case 1 (Ba-c) showed high intensity in the globus pallidus, thalamus, dentate nuclei, and subcortical white matter; CT scan of case 2 (Bd-g) showed symmetrical calcification at the basal ganglia, thalamus, dentate nuclei, and subcortical white matter; case 3 (Bh) showed calcification in the basal ganglia and thalamus; and case 4 (Bi-l) showed prominent calcification in the basal ganglia, thalamus, dentate nuclei, cerebellar vermis, subcortical white matter, and brainstem.

were observed, including gait disorder, bradykinesia, involuntary movements, or psychiatric disorders. A neurological examination revealed dysarthria, forced laughter, and a positive Huffman's sign. Her CT images (TCS, 43) showed severe calcification at the bilateral basal ganglia, thalamus, dentate nuclei, and subcortical white matter (Figures 2Bd-g). Unfortunately, her parents were unable to provide brain CT or blood samples.

Case 3. The patient who carried c.1969G $>C$ and c.782_783GC $>$ TT heterozygous variants was a 68 -year-old female from Northern China. She presented with 23 years of vertigo and had a previous history of cerebral infarction, cerebral hemorrhage, hypertension, and coronary heart disease. A neurological examination was unremarkable. Laboratory tests showed normal levels of serum calcium, phosphorus, and parathyroid hormone. Her brain CT reported symmetrical calcification at the basal ganglia, thalamus, dentate nucleus, and corona radiata though a complete series of images was not available (Figure 2Bh). Unfortunately, we could not examine her parents because they have both passed away.

Case 4. The 62-year-old man carrying the homozygous c.2033C > $G$ variant experienced disease onset at the age of 59, with slowly progressive gait unsteadiness, reduced up and down gaze and slurred speech. A neurological examination revealed mild dysarthria, bradykinesia, rigidity of the limbs, staggering in tandem gait, and poor pointing performance of the finger-to-nose test. No other abnormalities were found, including psychiatric disorder, seizure, memory disturbance, or involuntary movements. His CT images (TCS, 57) revealed prominent calcification in the basal ganglia, thalamus, dentate nuclei, cerebellar vermis, and subcortical white matter (Figures 2Bi-1). Extensive brainstem calcifications affecting the pons and mesencephalon were also observed. Moreover, cerebellar atrophy could also be witnessed in the CT images (Figures 2Bi-j). Unfortunately, brain CT and genetic screening of his parents were unavailable due to their death. 


\section{DISCUSSION}

Our previous study suggests that mutations of $M Y O R G$ are the main cause of autosomal recessive PFBC (Yao et al., 2018). To date, more than 50 variants associated with PFBC have been reported in different ethnic populations, further confirming the pathogenicity of MYORG mutations (Bauer et al., 2019; Westenberger et al., 2019). In this study, we identified four novel variants of MYORG including two missense variants and two nonsense variants in four sporadic cases. All of these variants were absent in any control individuals indicating that they were likely to be deleterious variants. The variants c. $442 \mathrm{C}>\mathrm{T}$ and c.972C > A both led to the premature termination codon (p.Q148* and p. $\left.\mathrm{Y} 324^{*}\right)$ and resulted in a truncated protein. The two missense variants, c.1969G >C, p. G657R and c.2033C > G, p. P678R, were both located in glycosyl hydrolase domain (aa 311-714) and the highly conserved regions, resulting in an amino acid substitution that was predicted to negatively affect protein function. According to the standards and guidelines for the interpretation of sequence variants, c. $442 \mathrm{C}>\mathrm{T}$ and $\mathrm{c} .972 \mathrm{C}>\mathrm{A}$ could be considered as pathogenic variants with the evidence of PVS1 + PM2 + $\mathrm{PM} 3+\mathrm{PP} 3$ and PVS1 + PM2 + PP3. Also, c.2033C > G could be considered as a likely pathogenic variant based on existing evidence of PM1 + PM2 + PM3 + PP3 + PP4. However, we could not confirm that it fits an autosomal recessive pattern due to lack of examination of the parents of case 2. Therefore, we classified this variant c.1969G $>C$ as "uncertain significance" at this time point based on the evidence of PM1 + PM2 + PP3.

The role of the MYORG protein (known as NET37 or KIAA1161) is largely unknown. Based on sequence analysis, MYORG is predicted to be a member of the glycosyl hydrolase 31 family, with a glycosidase function (Datta et al., 2009). Our previous study suggested that MYORG was specifically expressed in astrocytes, a key component of the neurovascular unit (NVU), and mutations of MYORG would result in dysfunction in NVU. MYORG protein contains a transmembrane domain at its $\mathrm{N}$-terminus and a family 31 glycosyl hydrolase domain at its C-terminus. The two missense variants in our patients were both located in the glycosidase domain, and the two nonsense variants would result in a truncated protein without glycosidase domain, suggesting that the region plays an important role. The four novel variants in our patients could induce loss of glycosidase activity and, thus, lead to PFBC. However, additional study is needed to clarify the relationship between this mutations and resulting protein functions.

The clinical manifestations and neuroimaging features significantly vary among $\mathrm{PFBC}$ patients with mutations in different pathogenic genes. In our four patients, the brain CT findings showed widespread and abundant calcifications, which is consistent with the neuroimaging features in PFBC patients with MYORG mutations as reported by Chen et al. (Chen et al., 2019; Chen et al., 2020). Compared with other pathogenic genes, brainstem calcifications affecting the pons and cerebellar atrophy could be the prominent features in MYORG mutation carriers. This seems to be an indicator of this genetic form (Grangeon et al., 2019; Kume et al., 2020). Extensive calcifications encompassing the pons and extending to the whole brainstem were observed in case 4 . However, the calcifications in the basal ganglia and the cerebellum could not be distinguished from individuals with mutations of other PFBC pathogenic genes.

Patients with MYORG mutations tend to display a homogeneous clinical spectrum, showing dysarthria, parkinsonism, gait disorder, and ataxia (Taglia et al., 2019). Our patients also displayed dysarthria and parkinsonism as the major symptoms. As reported, dysarthria was shown to be a common prominent feature in the majority of symptomatic cases with MYORG mutations compared with those with mutations in the dominantcausing genes (Ramos et al., 2019). Parkinsonism was another common feature in our patients with MYORG mutations shown in case 1 and case 4 . Parkinsonism with vertical nuclear gaze palsy was uncommon in MYORG mutation carriers, but occurred in case 4, thus, extending the phenotypic spectrum of MYORG-related PFBC. Parkinsonism with vertical nuclear gaze palsy was reported to be associated with pontine calcifications, which were also noticed in the brain CT of case 4 (Chelban et al., 2020). In our study, homozygous or truncating mutations of MYORG could be associated with a more severe phenotype, such as in case 1 and case 4 .

It is possible that we underdiagnosed the MYORG mutations in our PFBC cohort due to certain limitations. First, we did not detect duplications or deletions of the total or part of the MYORG gene in the cohort. We also could not recruit familial members of the patients with MYORG variants for the co-segregation analyses. However, some MYORG synonymous variants found in this study could influence splicing.

The clinical symptoms and neuroimaging characteristics identified by this study, could aid clinicians in orienting genetic testing for PFBC, combining with the recessive inheritance patterns. In conclusion, we reported four novel MYORG mutations in Chinese PFBC, expanding the genetic and phenotypic spectrum of this disease.

\section{DATA AVAILABILITY STATEMENT}

All of the data supporting the findings in this study are available upon reasonable request from the corresponding authors.

\section{ETHICS STATEMENT}

The studies involving human participants were reviewed and approved by the institutional review board at the First Affiliated Hospital of Fujian Medical University. The patients/participants provided their written informed consent to participate in this study. 


\section{AUTHOR CONTRIBUTIONS}

Y-HZ designed and conceptualized the study, generated, collected, analyzed, and interpreted the data, and designed and made the diagrams. B-WL generated and collected the data, drafted the manuscript, and designed and made the diagrams. H-ZS collected the data, provided technical support, recruited the patients, and supervised the study. X-XG provided technical support and recruited the patients. Y-LL and L-LL collected the data. W-JC: designed and concepted study, analysis and interpretation, recruited patients, and supervised study. MZ designed and conceptualized the study, recruited the patients, and supervised the study. X-PY designed and conceptualized the study, analyzed and interpreted the data, designed and made the diagrams, supervised the study, and critically revised the important intellectual content of the manuscript. The final version of the manuscript was approved by all authors.

\section{FUNDING}

This work has been supported by the grants U1905210 (W-JC), 82171841 (X-PY) and 81801129 (X-PY) from the National Natural Science Foundation of China; the grant 2019J02010

\section{REFERENCES}

Bauer, M., Rahat, D., Zisman, E., Tabach, Y., Lossos, A., Meiner, V., et al. (2019). MYORG Mutations: A Major Cause of Recessive Primary Familial Brain Calcification. Curr. Neurol. Neurosci. Rep. 19, 70. doi:10.1007/s11910-0190986-Z

Cen, Z., Chen, Y., Chen, S., Wang, H., Yang, D., Zhang, H., et al. (2020). Biallelic Loss-Of-Function Mutations in JAM2 Cause Primary Familial Brain Calcification. Brain 143, 491-502. doi:10.1093/brain/awz392

Chelban, V., Carecchio, M., Rea, G., Bowirrat, A., Kirmani, S., Magistrelli, L., et al. (2020). MYORG-Related Disease Is Associated with Central Pontine Calcifications and Atypical Parkinsonism. Neurol. Genet. 6, e399. doi:10.1212/nxg.0000000000000399

Chen, Y., Cen, Z., Chen, X., Wang, H., Chen, S., Yang, D., et al. (2020). MYORG Mutation Heterozygosity Is Associated with Brain Calcification. Mov Disord. 35, 679-686. doi:10.1002/mds. 27973

Chen, Y., Fu, F., Chen, S., Cen, Z., Tang, H., Huang, J., et al. (2019). Evaluation of MYORG Mutations as a Novel Cause of Primary Familial Brain Calcification. Mov Disord. 34, 291-297. doi:10.1002/mds.27582

Datta, K., Guan, T., and Gerace, L. (2009). NET37, a Nuclear Envelope Transmembrane Protein with Glycosidase Homology, Is Involved in Myoblast Differentiation. J. Biol. Chem. 284, 29666-29676. doi:10.1074/jbc.m109.034041

Forouhideh, Y., Müller, K., Ruf, W., Assi, M., Şeker, T., Tunca, C., et al. (2019). A Biallelic Mutation linksMYORGto Autosomal-Recessive Primary Familial Brain Calcification. Brain 142, e4. doi:10.1093/brain/awy343

Grangeon, L., Wallon, D., Charbonnier, C., Quenez, O., Richard, A. C., Rousseau, S., et al. (2019). Biallelic MYORG Mutation Carriers Exhibit Primary Brain Calcification with a Distinct Phenotype. Brain 142, 1573-1586. doi:10.1093/brain/awz095

Guo, X. X., Zou, X. H., Wang, C., Yao, X. P., Su, H. Z., Lai, L. L., et al. (2019). Spectrum of SLC20A2, PDGFRB, PDGFB, and XPR1 Mutations in a Large Cohort of Patients with Primary Familial Brain Calcification. Hum. Mutat. 40, 392-403. doi:10.1002/humu.23703

Keller, A., Westenberger, A., Sobrido, M. J., García-Murias, M., Domingo, A., Sears, R. L., et al. (2013). Mutations in the Gene Encoding PDGF-B Cause Brain Calcifications in Humans and Mice. Nat. Genet. 45, 1077-1082. doi:10.1038/ ng.2723
(W-JC) from the Natural Science Foundation of Fujian Province; the grant 2018QH2035 (MZ) from the Startup Fund for Scientific Research of Fujian Medical University; the Post-doctoral Startup Fund for Scientific Research of the First Affiliated Hospital of Fujian Medical University (MZ); and the Scientific Research Foundation for the introduction of talent of the First Affiliated Hospital of Fujian Medical University (MZ).

\section{ACKNOWLEDGMENTS}

We thank all the patients and their family members for participating in this study.

\section{SUPPLEMENTARY MATERIAL}

The Supplementary Material for this article can be found online at: https://www.frontiersin.org/articles/10.3389/fgene.2021.732389/ full\#supplementary-material

Supplementary Figure S1 | Identification of four previously reported variants in our study. (A-D) Sanger sequences of the previously reported variants and corresponding wildtype subjects.

Kume, K., Takata, T., Morino, H., Matsuda, Y., Ohsawa, R., Tada, Y., et al. (2020). The First Japanese Case of Primary Familial Brain Calcification Caused by an MYORG Variant. J. Hum. Genet. 65, 917-920. doi:10.1038/s10038-020-0779-x

Legati, A., Giovannini, D., Nicolas, G., López-Sánchez, U., Quintáns, B., Oliveira, J. R. M., et al. (2015). Mutations in XPR1 Cause Primary Familial Brain Calcification Associated with Altered Phosphate export. Nat. Genet. 47, 579-581. doi:10.1038/ng.3289

Manyam, B. V. (2005). What Is and what Is Not 'Fahr's Disease'. Parkinsonism Relat. Disord. 11, 73-80. doi:10.1016/j.parkreldis.2004.12.001

Nicolas, G., Charbonnier, C., Campion, D., and Veltman, J. A. (2018). Estimation of Minimal Disease Prevalence from Population Genomic Data: Application to Primary Familial Brain Calcification. Am. J. Med. Genet. 177, 68-74. doi:10.1002/ajmg.b.32605

Nicolas, G., Pottier, C., Charbonnier, C., Guyant-Maréchal, L., Le Ber, I., Pariente, J., et al. (2013). Phenotypic Spectrum of Probable and Genetically-Confirmed Idiopathic Basal Ganglia Calcification. Brain 136, 3395-3407. doi:10.1093/ brain/awt 255

Nicolas, G., Pottier, C., Maltête, D., Coutant, S., Rovelet-Lecrux, A., Legallic, S. et al. (2013). Mutation of the PDGFRB Gene as a Cause of Idiopathic Basal Ganglia Calcification. Neurology 80, 181-187. doi:10.1212/ wnl.0b013e31827ccf34

Ramos, E. M., Roca, A., Chumchim, N., Dokuru, D. R., Van Berlo, V., De Michele, G., et al. (2019). Primary Familial Brain Calcification Caused by a Novel Homozygous MYORG Mutation in a Consanguineous Italian Family. Neurogenetics 20, 99-102. doi:10.1007/s10048-019-00571-8

Richards, S., Aziz, N., Aziz, N., Bale, S., Bick, D., Das, S., et al. (2015). Standards and Guidelines for the Interpretation of Sequence Variants: a Joint Consensus Recommendation of the American College of Medical Genetics and Genomics and the Association for Molecular Pathology. Genet. Med. 17, 405-423. doi:10.1038/gim.2015.30

Taglia, I., Bonifati, V., Mignarri, A., Dotti, M. T., and Federico, A. (2015). Primary Familial Brain Calcification: Update on Molecular Genetics. Neurol. Sci. 36, 787-794. doi:10.1007/s10072-015-2110-8

Taglia, I., Kuipers, D. J. S., Breedveld, G. J., Mignarri, A., Dotti, M. T., Federico, A., et al. (2019). Primary Familial Brain Calcification Caused by MYORG Mutations in an Italian Family. Parkinsonism Relat. Disord. 67, 24-26. doi:10.1016/j.parkreldis.2019.09.021 
Wang, C., Li, Y., Shi, L., Ren, J., Patti, M., Wang, T., et al. (2012). Mutations in SLC20A2 Link Familial Idiopathic Basal Ganglia Calcification with Phosphate Homeostasis. Nat. Genet. 44, 254-256. doi:10.1038/ng.1077

Westenberger, A., Balck, A., and Klein, C. (2019). Primary Familial Brain Calcifications: Genetic and Clinical Update. Curr. Opin. Neurol. 32, 571-578. doi:10.1097/wco.0000000000000712

Yao, X.-P., Cheng, X., Wang, C., Zhao, M., Guo, X.-X., Su, H.-Z., et al. (2018). Biallelic Mutations in MYORG Cause Autosomal Recessive Primary Familial Brain Calcification. Neuron 98, 1116-1123. doi:10.1016/j.neuron.2018.05.037

Conflict of Interest: The authors declare that the research was conducted in the absence of any commercial or financial relationships that could be construed as a potential conflict of interest.
Publisher's Note: All claims expressed in this article are solely those of the authors and do not necessarily represent those of their affiliated organizations, or those of the publisher, the editors and the reviewers. Any product that may be evaluated in this article, or claim that may be made by its manufacturer, is not guaranteed or endorsed by the publisher.

Copyright $\odot 2021$ Zeng, Lin, Su, Guo, Li, Lai, Chen, Zhao and Yao. This is an openaccess article distributed under the terms of the Creative Commons Attribution License (CC BY). The use, distribution or reproduction in other forums is permitted, provided the original author $(s)$ and the copyright owner(s) are credited and that the original publication in this journal is cited, in accordance with accepted academic practice. No use, distribution or reproduction is permitted which does not comply with these terms. 